\title{
Localized fluorescence excitation in opaque media by time-reversed ultrasonically encoded (TRUE) optical focusing
}

Yuta Suzuki, Puxiang Lai, Xiao Xu, Lihong Wang

Yuta Suzuki, Puxiang Lai, Xiao Xu, Lihong Wang, "Localized fluorescence excitation in opaque media by time-reversed ultrasonically encoded (TRUE) optical focusing," Proc. SPIE 8943, Photons Plus Ultrasound: Imaging and Sensing 2014, 894358 (3 March 2014); doi: 10.1117/12.2037947

SPIE. Event: SPIE BiOS, 2014, San Francisco, California, United States 


\title{
Localized fluorescence excitation in opaque media by time-reversed ultrasonically encoded (TRUE) optical focusing
}

\author{
Yuta Suzuki ${ }^{*}$ Puxiang Lai, Xiao Xu, and Lihong Wang* \\ Optical Imaging Laboratory, Department of Biomedical Engineering, \\ Washington University in St. Louis, St. Louis, Missouri, 63130, USA
}

\begin{abstract}
To focus light beyond one transport mean free path, time-reversed ultrasonically encoded (TRUE) optical focusing has previously been implemented by both analog and digital devices. By allowing wavefront recording with finer resolution and larger aperture, the analog scheme, which uses photorefractive materials as the phase-conjugate mirror, generates a more complete set of time-reversed optical modes than the digital scheme. Here, we report the direct visualization of localized fluorescence excitation inside a turbid medium by photorefractive time reversal. Further, we imaged fluorescent targets embedded in a turbid phantom whose thickness was four transport mean free paths.
\end{abstract}

Keywords: optical focusing, ultrasound modulation, optical imaging, phase conjugation, time reversal, photorefractive material.

\section{Introduction}

Light has been an essential visualizing tool in biomedical studies due to its non-invasive nature. However, because of multiple photon scattering events, optical focusing in biological tissue by conventional lenses is limited to the shallow depth of one transport mean free path (typically $1 \mathrm{~mm}$ in human skin), which results in degraded imaging resolution beyond this limit. To overcome these light scattering effects, $\mathrm{Xu}$ et al. developed a technique named timereversed ultrasonically encoded (TRUE) optical focusing [1]. Fig. 1(a)-(b) show the two-staged principle of TRUE focusing. In the holographic recording stage (Fig. 1a), a focused ultrasonic field is applied to frequency-modulate the

(a)

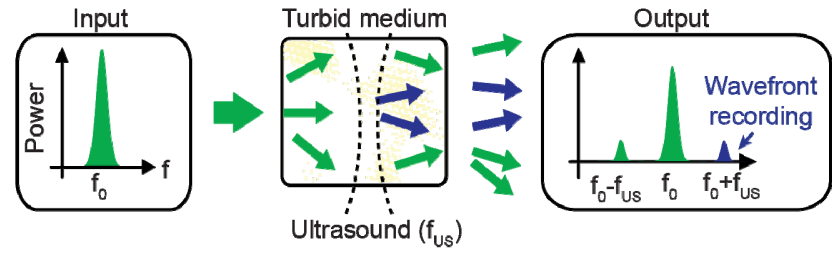

(b)

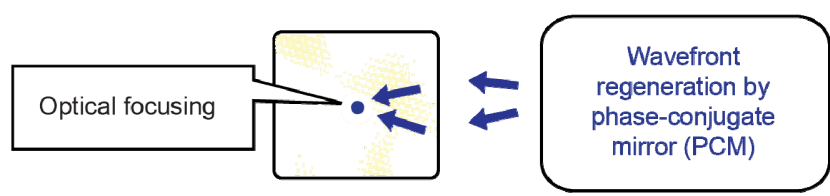

Figure 1. The principle of TRUE focusing. (a) Generation and recording of the ultrasonically encoded light inside a turbid medium. (b) Wavefront regeneration by a PCM and realization of TRUE focusing..

\footnotetext{
* Further author information

Yuta Suzuki, ysuzuk@wustl.edu, 1-314-935 9587

Lihong V. Wang, lhwang@,wustl.edu, 1-314- 9356152
}

Photons Plus Ultrasound: Imaging and Sensing 2014, edited by Alexander A. Oraevsky, Lihong V. Wang, Proc. of SPIE Vol. 8943, 894358 - @ 2014 SPIE · CCC code: 1605-7422/14/\$18 · doi: 10.1117/12.2037947 
incident sample beam (with a frequency of $f_{0}$ ) propagating in a turbid medium. As a result, the optical frequencies of the photons traversing the ultrasonic focal region are modulated by ultrasonic frequency $\left(f_{\text {US }}\right)$. Then the wavefront of the ultrasonically modulated, or encoded, optical component is selectively recorded outside the medium. The subsequent holographic readout stage (Fig. 1b) regenerates the recorded wavefront, which is the phase-conjugated beam of the encoded light. Due to the reversibility of light propagation, the phase-conjugated beam travels backwards and converges at the ultrasonic focus, thus forming an optical focus (TRUE focus) inside a turbid media.

The initial implementation of TRUE focusing was conducted using photorefractive material as an analogue PCM. Recently, digital implementation of the TRUE focusing system was reported, which uses a spatial light modulator (SLM) and a CMOS camera as the PCM [2,3]. Here, the wavefront of the encoded light is captured using digital holography; then, its phase-conjugated wavefront is reconstructed using an SLM. The main advantage of the digital implementation is a high optical gain, defined as the power ratio between the readout phase-conjugated beam and the recorded encoded light. While the holographic erasure in the photorefractive material typically limits the gain to less than 1, the digital PCM demonstrated a high gain of $\sim 5 \times 10^{5}$. Although this is an exciting advancement, the analog TRUE system still has attractive advantages over the digital version in other ways.

Firstly, the analog PCM can potentially generate a TRUE focus with high contrast, or a high peak-tobackground ratio (PBR). PBR has been used as an important measure of TRUE focusing, and is defined as the intensity ratio of the generated focus and the surrounding unfocused background light. It is known that the PBR is proportional to the number of independently controlled pixels of the PCM. While a state-of-art SLM has a pixel number of $\sim 2 \times 10^{6}$, an analog PCM resolves much finer spatial details, and can be equivalent to as large a number of controlled pixels as $10^{9}$ to $10^{11}$ [4]. Thus, analog PCM can potentially achieve TRUE focusing with a higher PBR. Secondly, the fast operational speed of the analog PCM is an important advantage for future biomedical applications. Digital TRUE focusing currently requires timescales on the order of seconds to generate a focus at a single position, while analog PCM can operate at speeds on the order of milliseconds, currently limited by the response time of the photorefractive material [1,5]. Lastly, the reported digital TRUE system requires a precise pixel-match between the sCMOS camera and the SLM, demanding day-to-day re-alignment to maintain quality consistency. On the other hand, the analog approach offers a simpler setup and a lower implementation cost than the digital approach, which are both desirable for many applications.

Here, by using an analog PCM for the TRUE optical system, we report the direct visualization of localized fluorescence excitation inside a turbid medium. Also, we show the fluorescent imaging results of fluorescent targets embedded in a turbid phantom whose thickness was four transport mean free paths.

\section{Optical setup}

Our TRUE system, using a photorefractive material as the analog PCM, is schematically illustrated in Fig. 2. In the recording stage, an ultrasonic beam is applied to the sample, and the sample beam is turned on to illuminate a turbid sample. The transmitted light through the sample is collected onto the PCM, where it is mixed with a flat reference

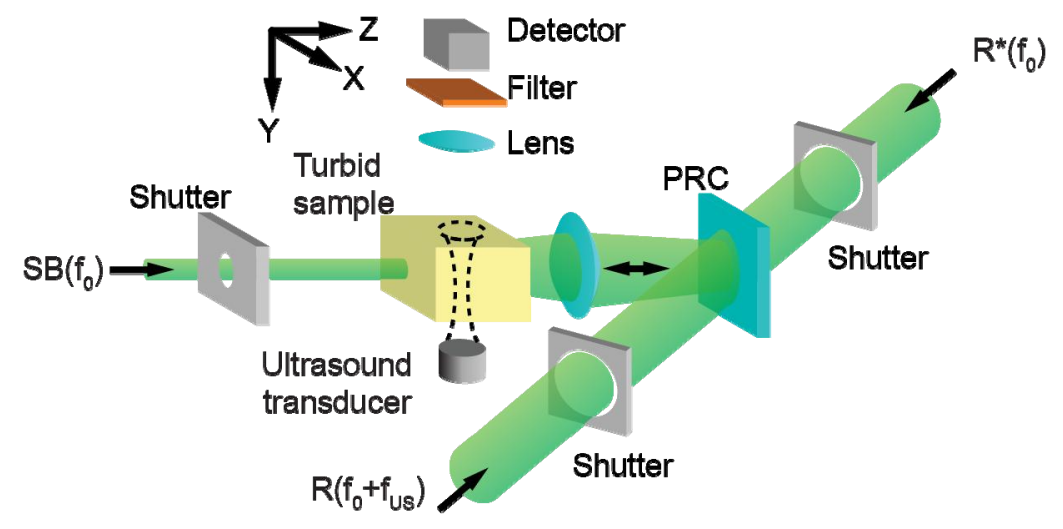

Figure 2. Schematic illustration of the TRUE system using photorefractive material as the analog PCM. 
sbeam R. By setting the frequency of the reference beam R to that of the ultrasonically upshifted light (encoded light), we selectively recorded a hologram of the encoded light inside the PCM through interference. At the end of the recording stage, both the sample beam (SB) and R were turned off. In the subsequent readout stage, the readout beam $\left(\mathrm{R}^{*}\right)$ was turned on to read out the hologram and to generate the time-reversed beam of the ultrasonically encoded light (TRUE light). The excited fluorescent signal by the TRUE light was detected by a detector mounted above the sample, after passing through properly chosen optical filters.

\section{Visualization of TRUE focus}

To directly observe the optical focusing in the turbid sample, we used a clear medium sandwiched between two turbid layers. The TRUE focus was targeted on a fluorescent bar placed in the clear medium, as shown in Fig. 3(a). The continuous-wave 3.5 MHz ultrasound with focal pressure of 1.1 MPa modulated light within the clear medium during the recording stage. An intensified charge coupled device (ICCD) imaged the excited fluorescence along the bar target after being filtered by a long pass filter during the readout stage. In this way, we evaluated the propagation of phaseconjugated light. We used a photorefractive polymer as the PCM because it can sustain a recorded hologram with a long

(a)

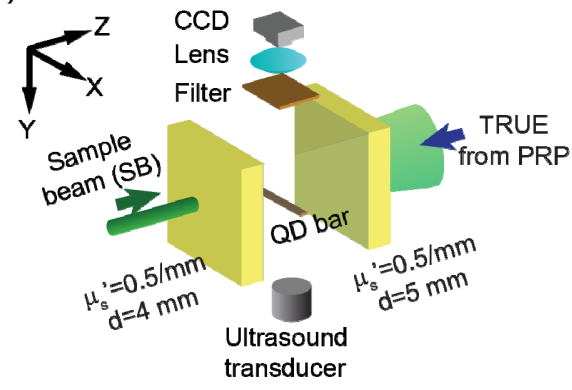

(c)

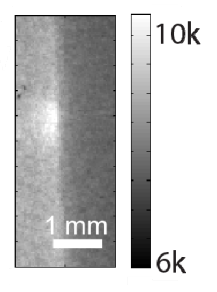

(d)

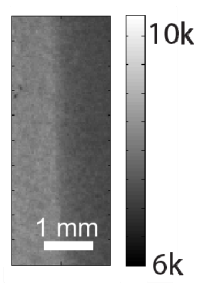

(b)

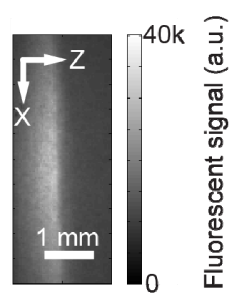

(e)

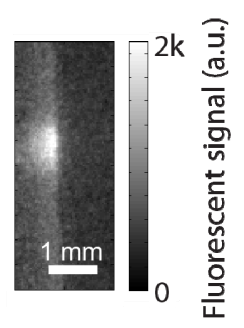

(f)

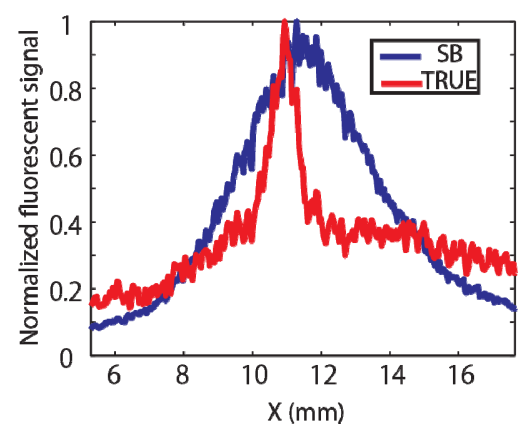

Figure 3. Visualization of TRUE focusing. (a) Experimental configuration. The excited fluorescence distribution on quantum-dot (QD) bar was captured by the charge-coupled device mounted above the sample. (b) Excited fluorescence by sample beam (SB). (c) The excited fluorescent light during the readout stage along the bar target. (d) The background fluorescent excitation when the ultrasound was turned off. (e) The net fluorescent excitation by the TRUE focus, obtained by subtracting the background from the excited fluorescent signal during the readout stage. (f) Comparison of the normalized fluorescence signal by SB excitation and TRUE focusing. 
persistency to increase the detected signal level at the ICCD [6]. The exposure time of the ICCD was set at $50 \mathrm{~ms}$. To reduce the effect of sample vibration by ultrasound, the back turbid layer was mounted separately using a different case from the first front two layers of the sample (the front turbid and the clear layers). The turbid layers used in this experiment had a reduced scattering coefficient $\mu_{\mathrm{s}}^{\prime}=5 \mathrm{~cm}^{-1}$, and the thicknesses of the front and back layers were $\sim 4 \mathrm{~mm}$ and $\sim 5 \mathrm{~mm}$, respectively. As a comparison to the TRUE fluorescent excitation, we also captured the fluorescent excitation by sample beam (SB) illumination.

The fluorescence excited by the SB is shown in Fig. 3(b), where we see a broad distribution of the fluorescent signal due to light diffusion by the front turbid layer. On the other hand, the TRUE readout generated a bright peak in the fluorescent excitation along the bar target, as seen in Fig. 3(c). Although we see a bright spot in Fig. 3(c), we noticed that the fluorescent signal is not solely from the TRUE focus, as we see some background excitation when the ultrasound was turned off, as shown in Fig. 3(d). By subtracting this background excitation (Fig. 3d) from the fluorescent excitation during the readout stage (Fig. 3c), we obtained the net fluorescent excitation by the TRUE focus, shown in Fig. 3(e). There, we more clearly see the excited bright spot by the TRUE focus.

Further, the cross sectional plot along the QD bar target in TRUE focusing is shown in Fig. 3(f), as well as the SB excitation. The measured focal width of the TRUE focus was $1 \mathrm{~mm}$ in terms of full-width at half maximum (FWHM), and the measured peak-to-background ratio (PBR) was $\sim 5$. Because our ultrasound focal volume was large enough to contain $\sim 5 \times 10^{7}$ speckles inside, the measured value of the PBR indicates that the number of controlled modes of our PCM was $\sim 0.25 \times 10^{9}$, which shows the advantage of our analog PCM over the digital scheme whose pixel number is currently $\sim 2 \times 10^{6}$.

\section{Fluorescent Imaging}

To demonstrate the capability of focused fluorescence imaging in turbid media, we prepared another experimental sample, as shown in Fig. 4(a). We used a $\mathrm{Bi}_{12} \mathrm{SiO}_{20}$ (BSO) crystal as the PCM for its faster response time. which is preferred for obtaining images. The ultrasonic field was generated by a 3.5-MHz transducer in burst mode with a duty cycle of $20 \%$, and the peak-to-peak focal pressure was decreased to $0.9 \mathrm{MPa}$ to further reduce the ultrasoundinduced sample speckle decorrelation. To ensure good acoustic coupling for ultrasonic wave propagation, two transparent layers sandwiched an 8-mm thick central turbid layer, and the bottom surface of the sample was immersed in water. At the mid- $z$ plane of this scattering layer, two objects $\left(2 \times 2 \times 1 \mathrm{~mm}^{3}\right.$ along $x, y$, and $z$, respectively), containing $2.8 \times 10^{-7} \mu \mathrm{M}$ polystyrene fluorescent microspheres ( $2 \mu \mathrm{m}$ of diameter, F8825, Invitrogen), were embedded $10 \mathrm{~mm}$ below the top surface, as shown in Fig. 4(b). We aligned the ultrasonic focus to the two pencil cores (graphite rods) in both $y$ and $z$ directions, along the plane that contains the two targets.

The fluorescent emission was sensed by an avalanche photodiode (APD, SPM3Q-T, Newport) after rejecting the background light at $532 \mathrm{~nm}$ using a long-pass filter (FGL590S, Thorlabs). To obtain a 1-D fluorescent image of the fluorescent targets, we scanned the sample along the $x$ direction with a step size of $0.2 \mathrm{~mm}$. At each position, we measured three fluorescent signals with the APD under different conditions: the SB intensity due to the incident signal beam during the holographic writing stage, the fluorescence excited by TRUE focusing, and the background signal when the frequency of the sample beam was detuned such that no hologram was formed. The excited fluorescent signals by TRUE focusing were subtracted with the background fluorescent signal to produce the net TRUE signal.

Fig. 4(c) shows the distribution of normalized fluorescent signal intensities as a function of sample position along the $x$ direction. In addition to the distribution of the TRUE signal, we plotted the fluorescent excitation by the SB as a reference. As we can see, the fluorescent excitation by the SB lacked the resolution to resolve the two closelypositioned fluorescent objects since they were excited by unfocused diffuse light. In contrast to the SB excitations, the fluorescent excitations by TRUE focusing revealed the size and the position of these two objects, due to a finer spatial resolution. From the obtained image, the two observed peaks had a FWHM of $2 \mathrm{~mm}$, and they were separated by $\sim 4 \mathrm{~mm}$, agreeing well with the arrangement of the fluorescent targets inside the sample. Moreover, the imaging resolution, evaluated from the Gaussian fit to the profile of the measured TRUE signal, was about $0.9 \mathrm{~mm}$, which is close to the focal width of the ultrasound beam used. Also, the measured resolution matches with the visualized excited fluorescence along the bar target in Fig. 3. This, once again, highlights that the spatial spread of the generated TRUE focus was 
determined by the ultrasonic focal volume, and also that our system was able to obtain fluorescent images in deep ( $\sim 4$ and $\sim 5$ transport mean free paths along $z$ and $y$, respectively) inside turbid media.

(a)

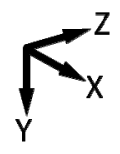

APD

Filter (b)

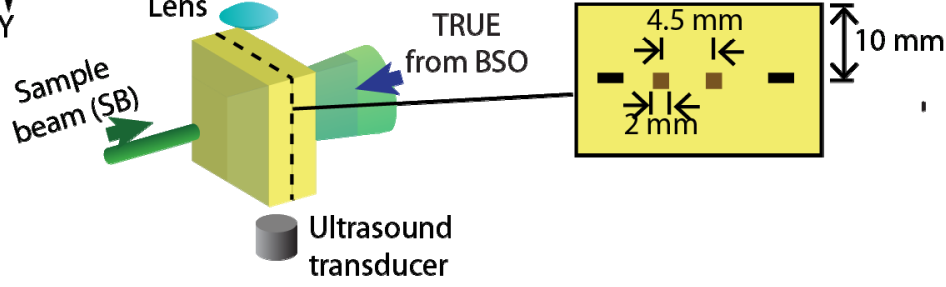

(c)

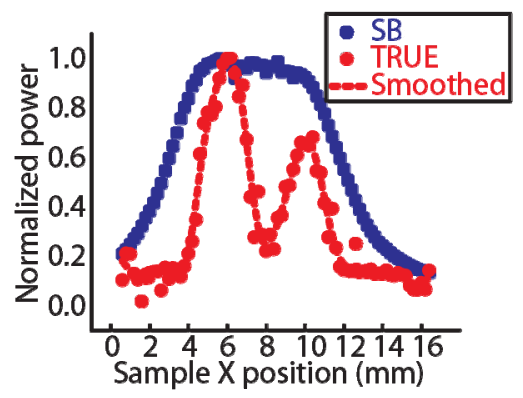

Figure 4. Fluorescent imaging inside a turbid medium by TRUE focusing. (a) Experimental configuration of imaging experiment. BSO, $\mathrm{Bi}_{12} \mathrm{SiO}_{20}$; APD, avalanche photo diode. (b) Schematic of the imaging plane set inside the turbid medium. Two fluorescent objects were used as the targets. (c) Obtained fluorescent image by TRUE focusing, shown together with fluorescent image by sample beam (SB).

\section{Discussion and Conclusions}

Delivery of a tightly focused light deep in turbid media (such as biological tissue) beyond the optical ballistic regime has seen intensive investigations. TRUE focusing has rapidly attracted much attention as it uniquely creates a virtual "guiding star" for optical focusing inside the turbid media.

It should be noted that the optical focusing investigated in our current study was only in two dimensions, because the use of continuous or long bursts of ultrasonic waves led to poor spatial resolution along the acoustic axis. However, by using short ultrasound pulses rather than long bursts, and by using a short pulsed laser, we can shrink the dimensions of the ultrasound-optical interaction region, which would improve the resolution along the acoustic axis and also the PBR of the generated TRUE focus.

Although the operational speed and penetration depth of our current TRUE system are limited by the sensitivity of our photorefractive materials, further improvements can be achieved by advanced filtering of the ultrasonically encoded light, as was done in the field of ultrasound-modulated tomography [7-9].

In summary, with TRUE systems that use a photorefractive polymer or a BSO crystal as the PCM, we have directly visualized the TRUE focus in $x$ direction by using the QD fluorescence dye. The focal spot widths of visualized TRUE foci corresponded well to the focal width of the used ultrasonic beam. Thus we confirmed a confined distribution of optical power inside the ultrasound focus by TRUE focusing, while mere sample beam illumination showed much broader distribution. From the observed TRUE focus, we estimated the number of controlled optical modes to be $\sim 0.25 \times 10^{9}$, which is much larger than the pixel number of current state-of-art SLMs $\left(\sim 2 \times 10^{6}\right)$, confirming the capability of analog PCM's to record and read out more independent optical modes than the digital PCM. Further, we demonstrated that the TRUE light can be used for focal fluorescence imaging deep inside turbid media with ultrasonically-determined 
spatial resolution. Since an analog approach of demonstrating TRUE focusing has the potential to achieve faster and finer TRUE focusing, the demonstrations of TRUE focal visualization and fluorescent imaging is an important milestone in this field.

\section{Acknowledgments}

We would like to thank Nitto Denko Technical (Oceanside, CA, USA) for providing the photorefractive polymer for this research. This work was sponsored in part by the National Academies Keck Futures Initiative grant IS 13 and National Institute of Health grants DP1 EB016986 (NIH Director's Pioneer Award), R01 EB000712 and U54 CA136398.

\section{References}

[1] Xu, X., Liu, H. L., and Wang, L. V., "Time-reversed ultrasonically encoded optical focusing into scattering media," Nature Photonics, 5, 154-157 (2011).

[2] Wang, Y. M., Judkewitz, B., DiMarzio, C. A., and Yang, C. H., "Deep-tissue focal fluorescence imaging with digitally time-reversed ultrasound-encoded light," Nature Communications, 3, 928-935 (2012).

[3] Si, K., Fiolka, R., and Cui, M., "Fluorescence imaging beyond the ballistic regime by ultrasound-pulse-guided digital phase conjugation," Nature Photonics, 6, 657-661 (2012).

[4] Lai, P., Suzuki, Y., Xu, X., and Wang, L. V., "Focused fluorescence excitation with time-reversed ultrasonically encoded light and imaging in thick scattering media," Laser Physics Letters, 10, 075604 (2013).

[5] Lai, P., Xu, X., Liu, H. L., Suzuki, Y., and Wang, L. V., "Reflection-mode time-reversed ultrasonically encoded optical focusing into turbid media," Journal of Biomedical Optics, 16, 080505 (2011).

[6] Suzuki, Y., Xu, X., Lai, P., and Wang, L. V., "Energy enhancement in time-reversed ultrasonically encoded optical focusing using a photorefractive polymer," Journal of Biomedical Optics, 17, 080507 (2012).

[7] Li, Y., Hemmer, P. L., Kim, C., Zhang, H., and Wang, L. V., "Detection of ultrasound-modulated diffuse photons using spectral-hole burning," Optics Express, 16, 14862 (2008).

[8] Murray, T. W., Sui, L., Maguluri, G., Roy, R. A., Nieva, A., Blonigen, F. J., and DiMarizo, C. A., "Detection of ultrasound-modulated photons in diffuse media using the photorefractive effect," Optics Letters, 29, 2509-2511 (2004).

[9] Rousseau, G., Blouin, A., and Monchalin, J.-P., "Ultrasound-modulated optical imaging using a high-power pulsed laser and a double-pass confocal Fabry-Perot interferometer," Optics Letters, 34, 3445-3447 (2009). 